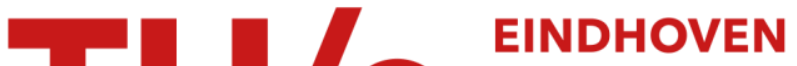 UNIVERSITY OF TECHNOLOGY
}

\section{Multipulse excitability in a semiconductor laser with optical injection}

\section{Citation for published version (APA):}

Wieczorek, S., Krauskopf, B., \& Lenstra, D. (2002). Multipulse excitability in a semiconductor laser with optical injection. Physical Review Letters, 88(6), 063901-1/4. [063901]. https://doi.org/10.1103/PhysRevLett.88.063901

DOI:

10.1103/PhysRevLett.88.063901

Document status and date:

Published: 01/01/2002

\section{Document Version:}

Publisher's PDF, also known as Version of Record (includes final page, issue and volume numbers)

\section{Please check the document version of this publication:}

- A submitted manuscript is the version of the article upon submission and before peer-review. There can be important differences between the submitted version and the official published version of record. People interested in the research are advised to contact the author for the final version of the publication, or visit the $\mathrm{DOI}$ to the publisher's website.

- The final author version and the galley proof are versions of the publication after peer review.

- The final published version features the final layout of the paper including the volume, issue and page numbers.

Link to publication

\section{General rights}

Copyright and moral rights for the publications made accessible in the public portal are retained by the authors and/or other copyright owners and it is a condition of accessing publications that users recognise and abide by the legal requirements associated with these rights.

- Users may download and print one copy of any publication from the public portal for the purpose of private study or research.

- You may not further distribute the material or use it for any profit-making activity or commercial gain

- You may freely distribute the URL identifying the publication in the public portal.

If the publication is distributed under the terms of Article 25fa of the Dutch Copyright Act, indicated by the "Taverne" license above, please follow below link for the End User Agreement:

www.tue.nl/taverne

Take down policy

If you believe that this document breaches copyright please contact us at:

openaccess@tue.nl

providing details and we will investigate your claim. 


\title{
Multipulse Excitability in a Semiconductor Laser with Optical Injection
}

\author{
Sebastian Wieczorek, ${ }^{1}$ Bernd Krauskopf,${ }^{2, *}$ and Daan Lenstra ${ }^{1}$ \\ ${ }^{1}$ Department of Physics and Astronomy, Vrije Universiteit Amsterdam, \\ De Boelelaan 1081, 1081 HV Amsterdam, The Netherlands \\ ${ }^{2}$ Department of Engineering Mathematics, University of Bristol, \\ Bristol BS8 1TR, United Kingdom \\ (Received 3 October 2001; published 25 January 2002)
}

\begin{abstract}
An optically injected semiconductor laser can produce excitable multipulses. Homoclinic bifurcation curves confine experimentally accessible regions in parameter space where the laser emits a certain number of pulses after being triggered from its steady state by a single perturbation. This phenomenon is organized by a generic codimension-two homoclinic bifurcation and should also be observable in other systems.
\end{abstract}

DOI: 10.1103/PhysRevLett.88.063901

A system is called excitable when it produces a large nonlinear response to a small but sufficiently large perturbation from its stable equilibrium. When perturbed above a certain threshold, an excitable system makes a large excursion in phase space leading to a large amplitude pulse. It then settles back to the stable equilibrium in what is called the refractory period. Here we use the example of an optically driven semiconductor laser to show that an excitable response to a single stimulus may have a form of not just a single pulse but of a certain fixed number of pulses. How many pulses are produced depends on the laser's operating conditions.

The notion of excitability was first introduced in biology to describe the spiking behavior of nerve cells [1] and later found in reaction-diffusion systems [2]. More recently different types of excitability were found in optical systems, ranging from nonlinear cavities with temperaturedependent absorption [3] to lasers with saturable absorber [4], optical feedback [5], and optical injection [6]. In lasers essentially two types of excitability are found [4]: the classic case of excitability on an invariant circle, and excitability in the vicinity of a homoclinic bifurcation, found first in lasers with saturable absorber [4] and then in multisection distributed feedback lasers [7]. All these examples of excitability were associated with a single-pulse response to a single perturbation. A double-pulse excitable response observed in a laser with optical feedback was explained as the result of noise on a two-dimensional model [5].

Our main result is that a multipulse response to a single perturbation is a natural and deterministic phenomenon. It is an example of excitability near a homoclinic bifurcation, but in this case near an $n$-homoclinic bifurcation, where the homoclinic orbit (to a saddle focus) closes up not after the first but after the $n$th global loop; see Figs. 2 and 4 below. The key element is a generic bifurcation structure of tongues formed by $n$-homoclinic bifurcation curves in parameter space. These tongues are organized by special points called Belyakov bifurcation points $[8,9]$, and inside each such tongue a different number of pulses will be triggered. We show multipulse excitability here
PACS numbers: 42.65.Sf, 05.45.-a, 42.55.Px

for the example of an optically injected semiconductor laser, but, in fact, this phenomenon may occur in any at least three-dimensional system with Belyakov bifurcation points. Other examples of systems with Belyakov points are an atmospheric circulation model [10], a tritrophic food chain model [11], and a reduced model of a multisection semiconductor laser [12].

We work with an optically injected single-mode semiconductor laser because it is a technologically important example of a forced nonlinear oscillator [13] for which astonishingly accurate experimental verification of various types of dynamics was demonstrated, both at local and global scale [14]. A single-mode class-B laser with optical injection is described well by the rate equations

$$
\begin{gathered}
\dot{E}=K+\left[\frac{1}{2}(1+i \alpha) n-i \omega\right] E, \\
\dot{n}=-2 \Gamma n-(1+2 B n)\left(|E|^{2}-1\right),
\end{gathered}
$$

which are a three-dimensional dynamical system for the complex electric field amplitude $E=E_{x}+i E_{y}$ and the population inversion $n$; see Ref. [13] for more details. The two experimental control parameters are the injected field rate $K$ and its detuning $\omega$ (measured in units of the characteristic relaxation oscillation frequency $\omega_{r}$ ) from the free-running laser frequency. We focus here on the case of a semiconductor laser, so that the self-modulation parameter $\alpha$ is larger than one, and we fix the laser parameters to the realistic values $\alpha=2, B=0.015$, and $\Gamma=0.035$.

When $K$ and $\omega$ are changed, the solutions of Eqs. (1) generally change as well. Qualitative changes of the system's dynamics, the so-called bifurcations, can be detected and continued in the two-dimensional $(K, \omega)$-plane, for example, with the package AUTO [15]. The resulting bifurcation curves form a bifurcation diagram by dividing the $(K, \omega)$-plane into regions with different dynamical behavior of the laser; see Ref. [13] for details of the bifurcation diagram of Eqs. (1).

Here we focus on the locking region inside which the laser operates at constant power and the injected light frequency. Locking is represented by a stable equilibrium in 
the three-dimensional phase space $\left(E_{x}, E_{y}, n\right)$. The locking region in the $(K, \omega)$-plane plotted in Fig. 1 is confined between the Hopf bifurcation curve $H$ and the saddlenode bifurcation curve $S N$. When the locking region is approached from above, the laser typically shows intensity oscillations at the relaxation oscillation frequency $\sim \omega_{R}$. At $H$ the corresponding stable periodic orbit disappears in a Hopf bifurcation, leading to a stable equilibrium, which is the locked state. When the locking region is approached from below, the laser produces oscillations at a frequency close to the detuning $\omega$. Close to $S N$ the flow on the corresponding periodic orbit slows down near the point where the saddle node will appear and then makes a quick excursion along the other part of the periodic orbit. At $S N$ two points, an attractor and a saddle focus, appear and the laser locks.

It was generally believed that in Eqs. (1) the saddle-node bifurcation always takes place on a periodic orbit. (This is also known as Adler's locking mechanism.) Here we show that this does not happen along the whole of the curve $S N$. As is also shown in Fig. 1 there are special points $A_{1}$ and $A_{2}$ where a homoclinic bifurcation curve labeled $h^{1}$ touches $S N$ (codimension-two saddle-node homoclinic points), forming what we call a homoclinic tooth. As a consequence, between $A_{1}$ and $A_{2}$ the saddle-node bifurcation does not take place on the periodic orbit. The same is true between $A_{3}$ and $A_{4}$ and at many more homoclinic teeth (not shown Fig. 1) that become smaller and smaller for larger $K$. In Eqs. (1) the size of the teeth depends crucially on the parameter $\alpha$ : they appear only for values of $\alpha>1$ and grow in size as $\alpha$ increases.

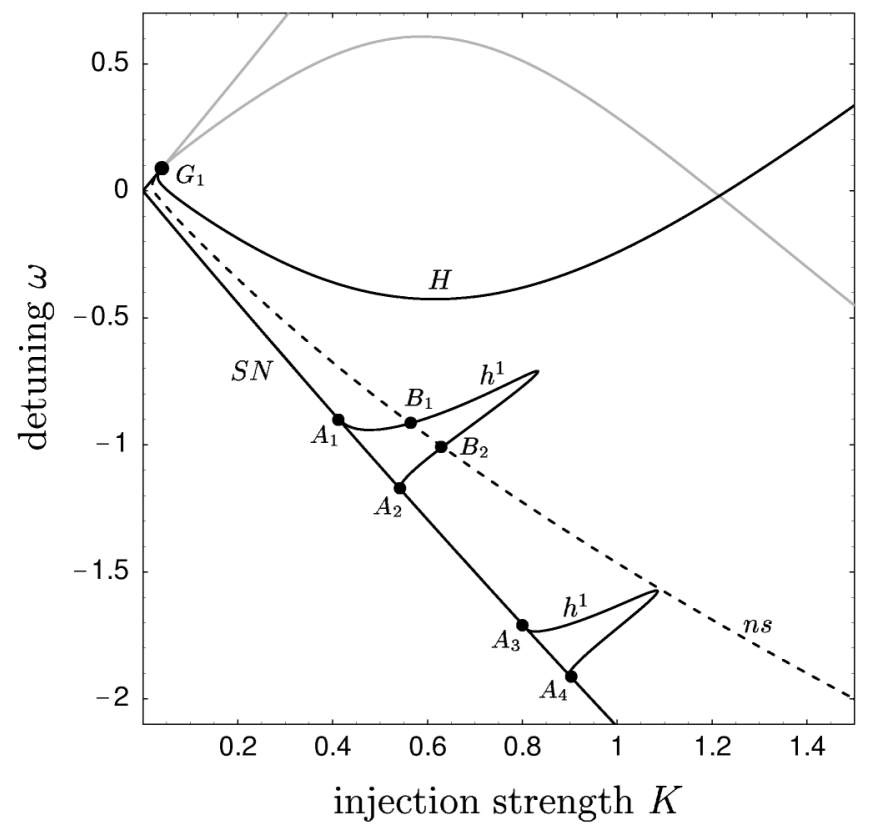

FIG. 1. The locking range of Eqs. (1) in the $(K, \omega)$-plane with two homoclinic teeth. In this and all figures of the $(K, \omega)$-plane, $\omega$ is in units of $\omega_{R}$ while $K$ is dimensionless. The gray curves correspond to subcritical bifurcations, along which no attractors bifurcate.
Near $h^{1}$ but outside a homoclinic tooth, the dynamics is as sketched in Fig. 2(a). On $h^{1}$ the one-dimensional unstable manifold $W^{u}$ of the saddle-focus equilibrium $s$ coalesces with the two-dimensional stable manifold $W^{s}$ of $s$, forming a one-homoclinic orbit; see Fig. 2(b). What the dynamics looks like inside the homoclinic tooth depends crucially on whether the stable or the unstable direction of the saddle focus is stronger [16]. The dashed curve $n s$ in Fig. 1 marks where the saddle focus is as attracting as repelling (neutral saddle). Along the parts of $h^{1}$ below $n s$ the attracting direction of the saddle focus is stronger and the homoclinic orbit bifurcates into an attracting periodic orbit as in Fig. 2(c1). This is often called a simple Shil'nikov bifurcation. The two-dimensional stable manifold $W^{s}$ forms the boundary between the basin of the locked state $a$ and that of the attracting periodic orbit. On the other hand, when $h^{1}$ is crossed above $n s$, then the unstable manifold makes two loops above $s$ and converges to the stable equilibrium $a$ as in Fig. 2(c2). This is often called a chaotic Shil'nikov bifurcation, because it implies the existence of an infinite number of saddle periodic orbits of different periods close to $h^{1}$. Furthermore, there are homoclinic bifurcation curves $h^{n}$ for any $n=1,2,3, \ldots$ of $n$-homoclinic orbits to the saddle focus $s$. They lie inside and near $h^{1}$, creating a cascade of bifurcations that is not entirely understood yet [9]. The codimension-two Belyakov points $B_{1}$ and $B_{2}$ are special as they form the boundary on $h^{1}$ between these types of Shil'nikov bifurcations.

We now reveal the homoclinic bifurcation structure associated with $B_{1}$ and $B_{2}$ in Eqs. (1) and show how this introduces regions in the $(K, \omega)$-plane with multipulse excitability. In Fig. 3(a) is plotted an enlargement of the biggest homoclinic tooth with some of the additional curves $h^{n}$ (computed with the HOMCONT part of AUTO [15]). There are two types of such curves: those that accumulate in the form of tongues at $B_{1}$ above $n s$, and those that cross the neutral-saddle line $n s$ [8]. We find that many $h^{n}$ tongues cross $n s$ and extend to the region of simple Shil'nikov bifurcation. Every intersection point of such a homoclinic bifurcation curve with $n s$ is a new Belyakov point. Tongues that approach $S N$ grow larger and are, in fact, experimentally accessible. (The experimental resolution is $\approx 100 \mathrm{MHz}$, while the detuning $\omega$ is in units of $\omega_{R} \approx 30 \mathrm{rad} / \mathrm{s}[14]$.) Some of the curves $h^{n}$ even connect to the curve $S N$ and create new segments where the
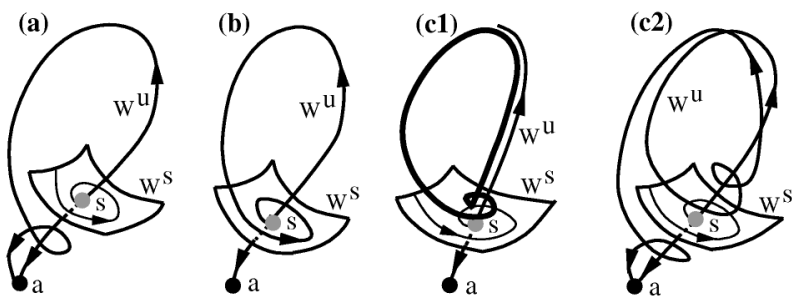

FIG. 2. Sketches of the phase portraits before (a), at (b), and just after (c1),(c2) the Shil'nikov bifurcation along $h^{1}$ for the simple case (c1) and the chaotic case (c2). 

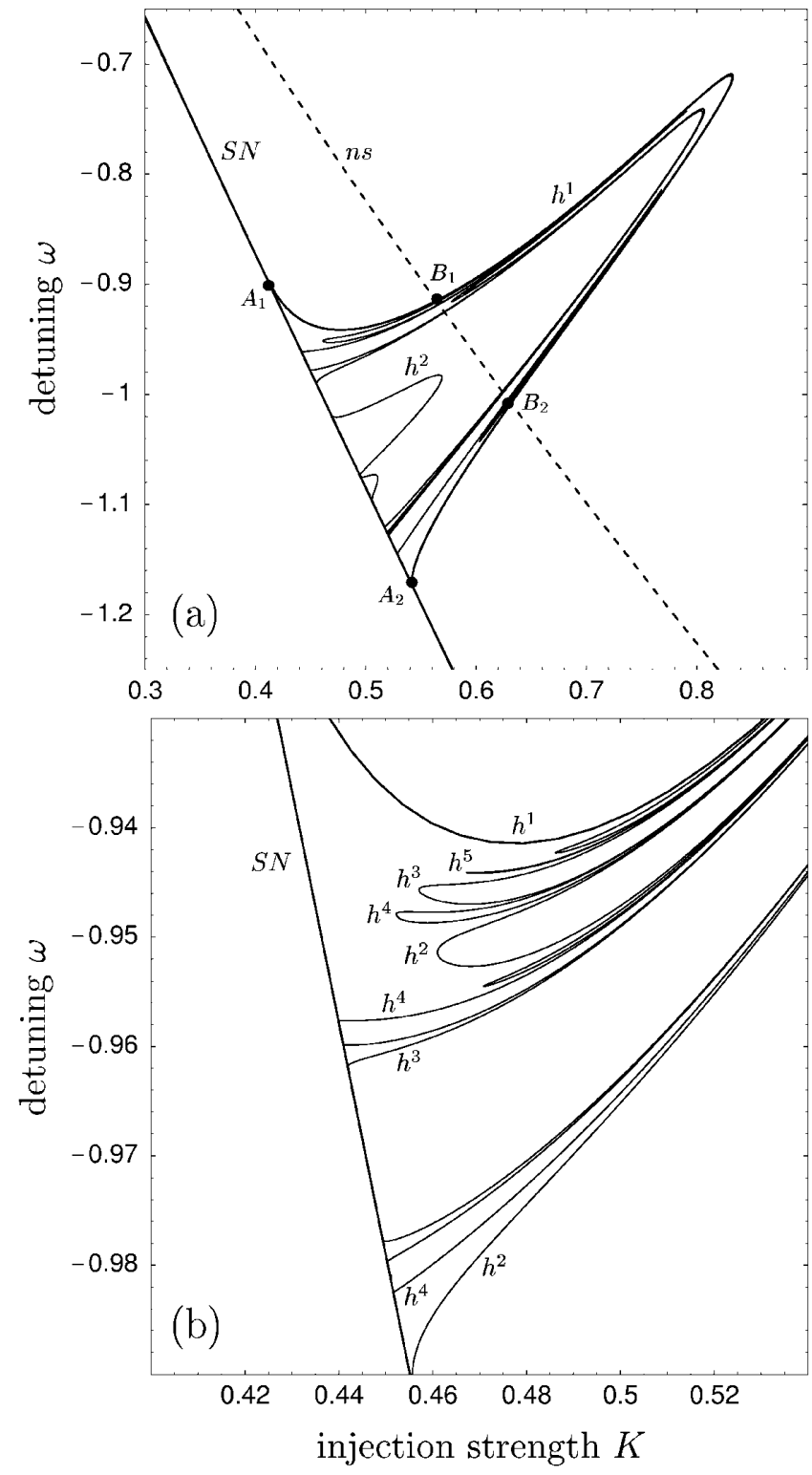

FIG. 3. Successive enlargements near the bigger homoclinic tooth in Fig. 1 showing tongues of multipulse excitability.

saddle-node bifurcation takes place on a limit cycle with $n$ loops. Figure 3(b) shows a further enlargement near $S N$ with more $n$-homoclinic bifurcation curves. For parameters between tongues and below $n s$ the laser is either locked to the input signal or shows self-pulsations.

For values of $K$ and $\omega$ inside each tongue bounded by $h^{n}$ and below $n s$ we find multipulse excitability. Figure 4 shows this for $(K, \omega)$ inside the tongues bounded by the curves $h^{1}, h^{2}, h^{3}$, and $h^{4}$ from Fig. 3(b). Plotted are the unstable manifolds $W^{u}$ of the saddle (left column) and the intensity response to a single small perturbation (right column), calculated with DSTOOL [17]. The mechanism of multipulse excitability is the following. The upper branch of the unstable manifold $W^{u}$ of the saddle makes $n$ loops above the stable manifold $W^{s}$ and then dives under $W^{s}$ to end up at the stable equilibrium; see Fig. 4 (left column). A single very small perturbation of the stable locked state
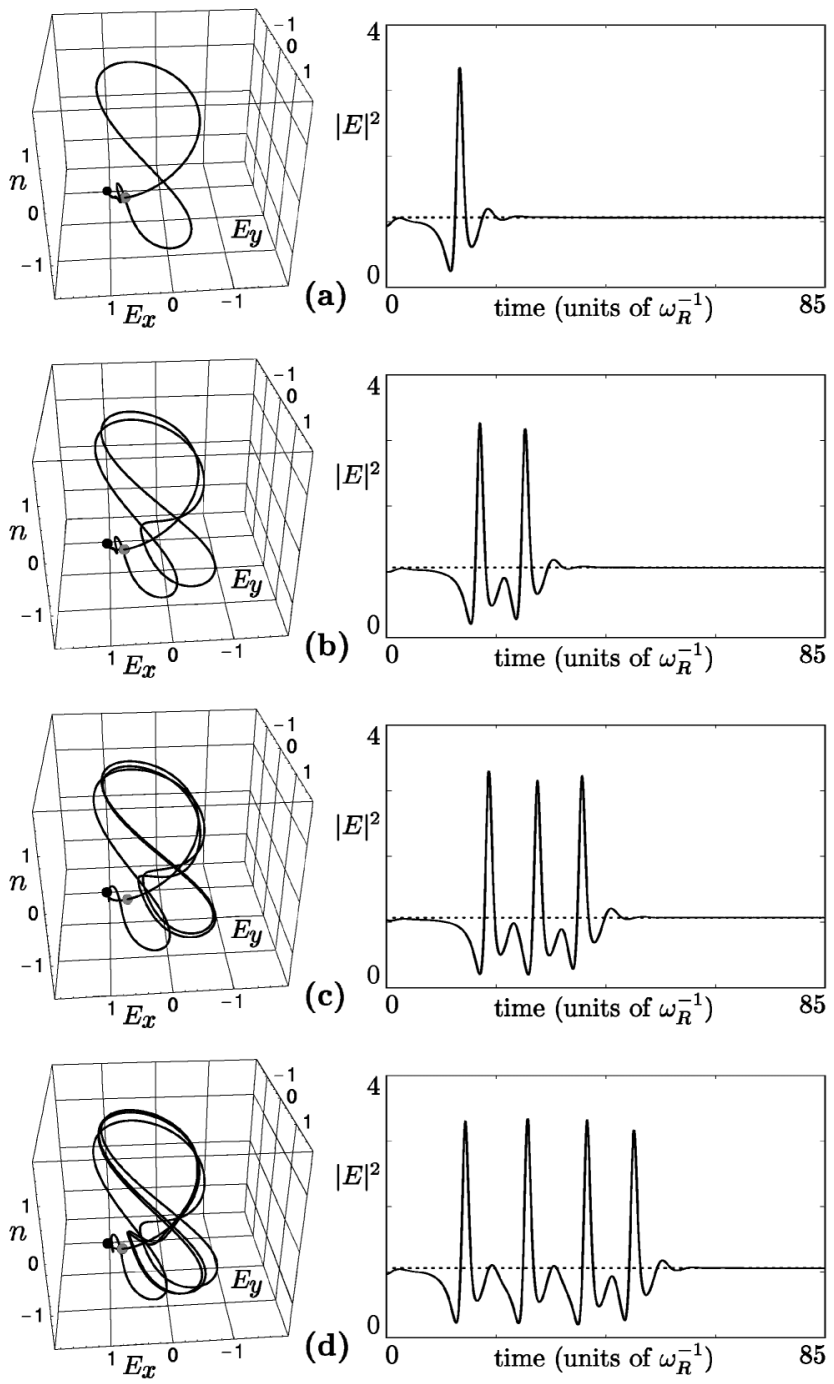

FIG. 4. Multipulse excitability in $(E, n)$-space (left column) and as intensity response to a single perturbation (right column) for $(K, \omega)$ from inside $h^{1}$ (a), $h^{2}$ (b), $h^{3}$ (c), and $h^{4}$ (d). From (a) to $(\mathrm{d})(K, \omega)$ takes the values $(0.45,-0.93),(0.472,-0.98)$, $(0.48,-0.97)$, and $(0.455,-0.95725)$.

results in the immediate return to the locked state. However, if the perturbation is strong enough to kick the system to the other side of $W^{s}$, then it follows the upper branch of $W^{s}$ and, hence, produces an $n$-pulse response; see Fig. 4 (right column). The threshold for excitability is given by $W^{s}$. Since, near the saddle, this is roughly given by the distance between the saddle and the attractor, this threshold becomes smaller closer to the curve $S N$. We finally point out that for certain parameter values near $h^{1}$ above $n s$ the excitable response can be chaotic and unpredictable, because the trajectory may wander between a huge number of coexisting unstable orbits before it settles back to the stable equilibrium.

In conclusion, we presented a deterministic mechanism for multipulse excitability that appears to be experimentally accessible in a real laser. We identified and described codimension-two homoclinic Belyakov bifurcations and an ensuing cascade of $n$-homoclinic bifurcation tongues as 
responsible for this phenomenon. Therefore, one should also find this type of multipulse excitability in other systems from applications in parameter regions with Belyakov points. In a real system there is always some noise so that some pulses may be triggered while the system follows the unstable manifold before the locked state is reached again [5]. As a result, a single perturbation inside an $h^{n}$ tongue may occasionally result in more than $n$ pulses as a response. Furthermore, close to $S N$ (spontaneous emission) noise itself may be enough to trigger pulses. Finally, we mention that the phenomenon of coherence resonance must be expected: a minimum of the jitter of a noisetriggered pulse train for a particular noise level [4]. How this general phenomenon manifests itself in the presence of multipulse excitability remains an interesting question for future research.

The research of S.W. was supported by the Foundation for Fundamental Research on Matter (FOM), which is financially supported by the Netherlands Organization for Scientific Research (NWO). B. K. is supported by the EPSRC.

*Corresponding author.

Email address: B.Krauskopf@bristol.ac.uk

[1] J. D. Murray, Mathematical Biology (Springer, New York, 1990).

[2] S. Grill, V.S. Zykov, and A.H. Cohen, Excitable Oscillators as Models for Central Pattern Generators, Series on Stability, Vibration and Control of Systems, Series B (World Scientific, Singapore, 1997).
[3] W. Lu, D. Yu, and R. G. Harrison, Phys. Rev. A 58, R809 (1998).

[4] J. L. A. Dubbeldam, B. Krauskopf, and Daan Lenstra, Phys. Rev. E 60, 6580 (1999).

[5] A. M. Yacomotti, M.C. Eguia, J. Aliaga, O. E. Martinez, G. B. Mindlin, and A. Lipsich, Phys. Rev. Lett. 83, 292 (1999).

[6] J. R. Tredicce, in Fundamental Issues of Nonlinear Laser Dynamics, edited by B. Krauskopf and D. Lenstra, AIP Conf. Proc. No. 548 (AIP, Melville, NY, 2000), p. 238.

[7] H. J. Wünsche, O. Brox, M. Radziunas, and F. Henneberger, Phys. Rev. Lett. 88, 023901 (2002).

[8] L. Belyakov, Mat. Zam. 36, 838 (1984).

[9] S. V. Gonchenko, D. V. Turayev, P. Gaspard, and G. Nicolis, Nonlinearity 10, 409 (1997).

[10] A. Shil'nikov, G. Nicolis, and C. Nicolis, Int. J. Bifurcation Chaos Appl. Sci. Eng. 6, 1701 (1995).

[11] Yu. A. Kuznetsov, O. de Feo, and S. Rinaldi, SIAM J. Appl. Math. (to be published).

[12] J. Sieber, "Numerical bifurcation for multisection semiconductor lasers" (to be published).

[13] S. Wieczorek, B. Krauskopf, and D. Lenstra, Opt. Commun. 4172, 279 (1999).

[14] S. Wieczorek, T. B. Simpson, B. Krauskopf, and D. Lenstra, "Global quantitative predictions of complex laser dynamics" (to be published).

[15] E. Doedel, T. Fairgrieve, B. Sandstede, A. Champneys, Yu. Kuznetsov, and X. Wang, AUTO 97, http:// indy.cs.concordia.ca/auto/main.html.

[16] Yu. A. Kuznetsov, Elements of Applied Bifurcation Theory (Springer, New York, 1995).

[17] A. Back, J. Guckenheimer, M. R. Myers, F. J. Wicklin, and P. A. Worfolk, Notices Am. Math. Soc. 39, 303 (1992). 